\title{
Geospatial Physicalization in Geography Education
}

\section{Lynn Moorman $^{\mathrm{a} *}$, Hessam Djavaherpour ${ }^{\mathrm{b}}$, Katayoon Etemad ${ }^{\mathrm{b}}$, and Faramarz Samavati ${ }^{\mathrm{b}}$}

${ }^{a}$ Earth and Environmental Sciences, Mount Royal University, Calgary, Canada;

${ }^{b}$ Department of Computer Science, University of Calgary, Calgary, Canada

lmoorman@mtroyal.ca*, \{hessam.djavaherpour, ketemad, samavati\}@ucalgary.ca

*Corresponding author

\begin{abstract}
A novel method to create a physicalization of Digital Earth resulted a new type of analogue and tactile geographic information system (GIS). The model was tested by students in Australia, who shared insights into how the model supported their learning about the concept of "overlay" while providing an interesting and engaging learning platform. Observations suggest the model promoted collaborative learning, and provided a bridge between paper maps and digital GIS. This study offers insight into preferences of school children for physicalization models in geography education and contributes to an understanding of children's spatial thinking and understanding of the overlay concept.
\end{abstract}

Keywords: geography education, visualization, spatial thinking, physicalization, analogue model, GIS, Digital Earth

Subject classification codes: geovisualization, map overlay, models, geography education, student attitudes

\section{Introduction}

Analogue models of Earth, traditionally maps and globes, are mainstays in geography and science education for visualizing information and systems that exist on scales that cannot be explored otherwise (Kastens and Rivet 2010). Hands-on models serve not only to display information, but have the potential to also facilitate inquiry and help develop a conceptual 
understanding (Kastens and Rivet 2008). Digital models, in the form of geographic information systems (GIS) and Digital Earth (DE), allow for multiple layers of data to be visualized and analysed upon an Earth model that can be dynamically viewed from multiple perspectives, including the full globe. In the past two decades, advances in technology have improved the access and user interface of GIS and DE to enable non-professionals to use digital technologies for geographic analysis, however, a consistent and fundamental issue for geography education has been the lack of understanding or ability to read the data, and understand relationships between datasets (Baker 2002). Of particular concern is the concept of overlay: the idea of extracting information from multiple layers at once (Baker et al. 2015; Golledge, March, and Battersby 2008). While the overlay process itself is straightforward in a digital environment, developing a strong conceptual understanding of the process and constituent information is fundamental to ensuring the appropriate data are used and to make sense of the results. Until now, there have been few options for a middle ground from flat map or globe to GIS, a critical step in the scaffolding from understanding map representation to the overlay analysis of layers of data enabled in a digital environment. Anecdotally, basic analogue GIS has been attempted, often consisting of spatial (map) layers on individual transparencies, to help in the transition to the digital environment, but the literature demonstrates a paucity of effective analogue models allowing students a tactile experience of manipulating layers on their own, in a true Earth-like model.

The geovisualization work of Djavaherpour, Mahdavi-Amiri, and Samavati (2017) transforms a Digital Earth model and chosen datasets into physical models that can serve as an analogue GIS. Using a Discrete Global Grid System (DGGS) and digital fabrication techniques, physical models of parts of the Earth's surface are created, accurate to the geoid with realistic Earth curvature and geometry (but not topography or surface relief). Multiple 
layers of data can then be attached on the model, allowing for hands-on inquiry between and among datasets (Fig. 1).
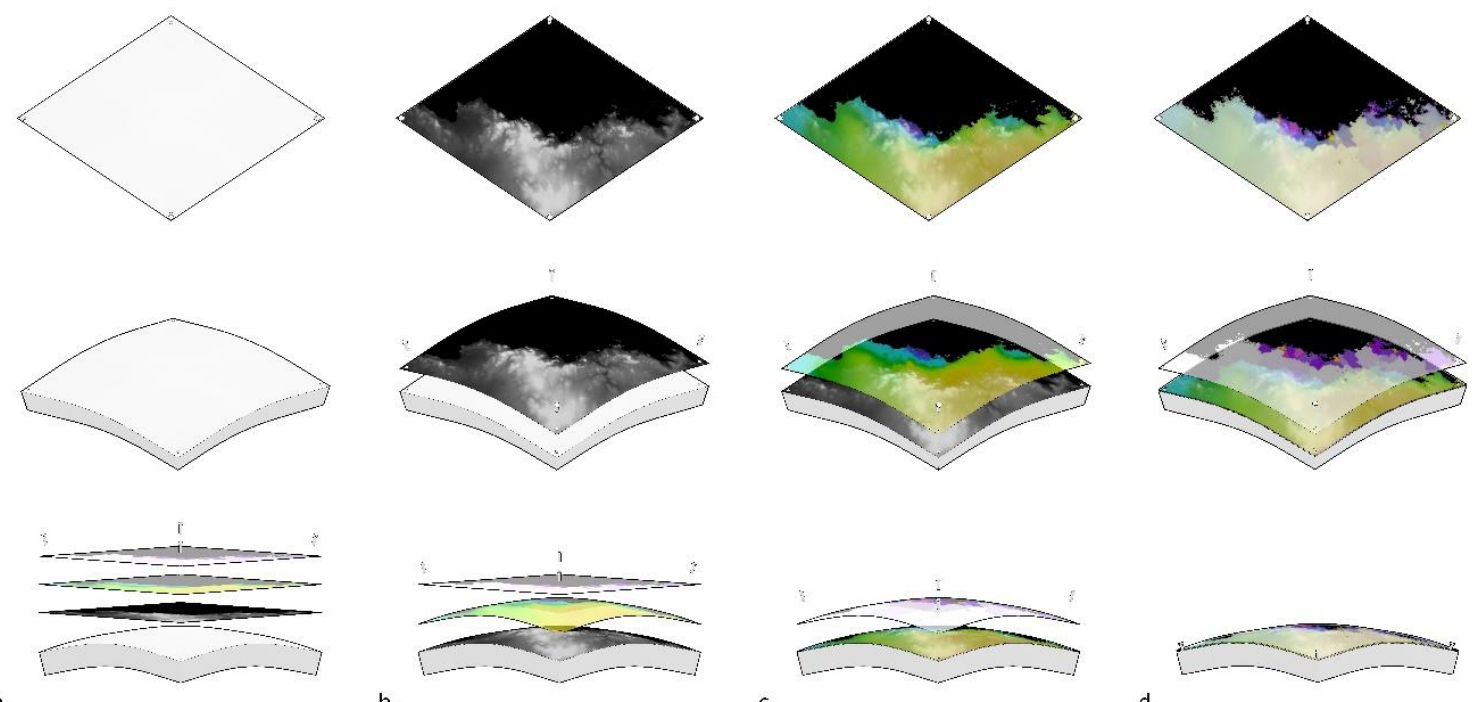

d.

Figure 1. Using a Discrete Global Grid System (DGGS) and digital fabrication techniques, physical models of parts of the Earth's surface are created, accurate to the geoid with realistic curvature and geometry. Multiple transparent layers of data can then be attached on the model, allowing for hands-on inquiry between and among datasets. (a) Base model and three layers ready to be attached, (b) 'elevation' data attached to the model, (c) and (d) transparent layers overlaid on 'elevation'.

While research in the fields of visualization and geographic technology typically focuses on technological advances, this work seeks to understand how that technology can be used most effectively for geographic education, specifically exploring the feasibility of using these analogue models for teaching geographic concepts. Feedback from end-users of the model will also inform the types of future pedagogic resources or strategies required by educators to support student learning with such physical models. This informed knowledge is aligned with the definition of Mishra and Koehler's (2006) technological pedagogical knowledge (TPK), the understanding of how teaching and learning can be affected by the use of specific technologies, and awareness of the best teaching practices to ensure appropriate 
learning. (Mishra and Koehler 2006). A study of students in Australia, ranging from 12-17 years of age, was conducted with the models to explore the following research questions:

(1) How did the students learn best with the model, particularly in the construct of overlay?

(2) What was the student experience using the model? and,

(3) How well suited is the model to a K-12 classroom?

This work contributes to the literature on geographic education and educational technology, particularly in the realm of spatial thinking and expressed dynamic models (Rivet and Kastens 2012), and provides important insights into a growing and increasingly important population for visualization and physicalization research and commercialization. Rarely are students' views and attitudes on the usability of a model considered (Gray et al. 2011). This study explored the student perspective and was conducted in a situated, authentic learning experience.

\section{Background}

\section{Geography Education}

Representations of Earth are the currency of geography, and are critical to teaching about location, geographic concepts and Earth processes. In terms of geography education, it is important that students have access to multiple representations of the world to build comprehensive and robust mental models (Jansen and Dragicevic 2013). If students are exposed to limited types of representations, their understanding is reified, and static, subject to the distortions and cartographer bias evident in those representations (Liben and Downs 
1989). Analogue models are frequently used in Earth Science education (Rivet and Kastens 2012), however many models are used by the instructor as a form of class demonstration, or they "tell the concept" rather than support development of conceptual understanding (Rivet and Kastens 2012, 715). The most common forms of Earth representations in K-12 classrooms are globes and maps, however, maps have inherent projection based distortions, and are a flat surface at any scale, while globes are limited in the scale of information that can be presented. The concept of "map projection" is a difficult concept for students of all ages to learn (Greco 2018). The projection and scale issues can be mitigated with digital dynamic globes, which allow the user to change scale to see large scale detail in small areas and then zoom out to see the Earth in its spherical form. However there are other challenges: the data that can be depicted are still limited for the education community, accessing digital platforms and content for an entire class at once is not always reliable in a K-12 environment, and collaborative learning opportunities may be limited or difficult with one person controlling the perspective.

A second concern around Earth representation has to do with the attribute data, or description of "what is there." Thematic maps display that information, for example, the country names on a typical classroom map, the types of land cover, or population density. To geographers, however, the real power is in determining and understanding the relationships between multiple thematic data. This can be facilitated digitally, through the visualization and analytic capability of GIS, but adopting this digital technology and data into middle level K-12 classrooms incurs challenges, including students not understanding the data (Baker 2002), struggling to make sense of the relationships between multiple layers (Golledge, Marsh, and Battersby 2008), or weak critical spatial thinking due to a focus on learning the software instead of focusing on the data content (Bearman et al. 2016). Currently there is a 
lack of bridging materials to support learners' progression from the tactile but distorted flat maps to a digital environment. This gap is an opportunity to promote active learning about data and introduce critical spatial thinking.

In the American National Geography Standards, Geography for Life, the Essential Element 1 (The World in Spatial Terms) and the first three standards all focus on fundamental skills of making meaning from geographic representations, using mental maps, and analysing spatial information, respectively (Gallagher and Downs 2012). Understanding how learners progress in their geospatial learning is a research priority (Recommendation 8) identified in the Road Map for 21st Century Geography (Bednarz, Heffron, and Huynh 2013), and formalized in the research agenda for geospatial technologies and learning (Baker at al. 2015). The agenda states "we have little information or guidance on best practices for how younger students learn GIS and other GST" (Geospatial Technologies) (Baker at al. 2015, 122).

\section{Geovisualization}

Harnessing the analytic power of a GIS, while keeping proper reference to the Earth's geoid, Digital Earth (DE) is a 3D digital representation of the Earth used as a reference model for the integration, management, visualization, and analysis of geospatial data (Mahdavi-Amiri, Alderson, and Samavati 2015). The most common approach to DE hierarchically divides the Earth into highly regular cells in different resolutions (a Discrete Global Grid System, i.e.,

DGGS). These cells are indexed to assign and retrieve geospatial datasets that can be "layered" on their appropriate locations and subsequently explored and analyzed (MahdaviAmiri, Samavati, and Peterson 2015; Alderson at al. 2020). 


\section{The Physicalization of Visualizations}

As viewing and exploring complex datasets requires modalities beyond screen-based interactions, data physicalization has recently emerged in the data visualization community (Jansen and Dragicevic 2013). A physical data visualization, or data physicalization, is a physical model that encodes data into its geometry and helps users understand and explore the data. Recent advances in digital fabrication and tangible interfaces have opened up various research possibilities in this field. Dadkhahfard et al. (2018) present a method for creating a dynamic visualization of geospatial data on a physical globe by using a piecewise curved-display and multi-projector setup. Examples of physical visualizations of geospatial datasets include spinning globes, physical terrain models, prism maps, and interactive dynamic displays (see dataphys.org/list/tag/cartographic). Compared with virtual or display models, concrete physical visualizations can be handled and controlled more easily, can improve users' cognition, and allow for more natural user interaction (Taher et al. 2015). By conducting a user study, Jansen, Dragicevic, and Fekete (2013) showed that a 3D physicalization improves the efficiency of users' information retrieval over on-screen visualization. The work of Stusak, Schwarz, and Butz (2015) explored the benefits of physicalization regarding memorability over digital-only models. According to the findings of their user study, participants who used the physical visualization remembered significantly more information within two weeks. Stusak, Hobe, and Butz $(2016,98)$ showed that "The better the mind can grasp the information behind a physical visualization, the more will grasping with the hands help." Recognizing the benefits of physical models, Horowitz and Schultz (2014) created study materials for lunar and planetary terrains and small space bodies. The authors claim that using both vision and manipulation of objects generates a 
tactile consciousness that often brings new elements into view for evaluation, which would not be detectable using digital models alone.

The work of Jansen et al. (2015) emphasized that to make a physicalization effective, we need to know which physical representation best engages the audience, how to encode variables in physical form, and how to support interactivity. This research seeks to answer those questions with a Digital Earth physicalization.

\section{Materials and Methods}

Two physical Earth models were created (described in detail below) and a study was conducted with four classes of students in Australia to explore the research questions. The research was conducted in the participants' regular classrooms to maintain a natural learning environment, and the teachers helped to introduce and run the exercises. The work was done in one class period for each group of participants. Data included information collected about the participant background, learning preference, and experience, their answers to specific tasks with the model and their responses to generally working with the model, in addition to researcher and teacher observations of how they used it. A mixed-methods analysis was employed to explore the ability of the students to use the model in answering geographic questions and supporting geographic inquiry, and to derive the thematic constituents of the students' effective response to the model to see if and how they "liked" working with it and whether they felt it supported their learning.

\section{Model}

For this study, two models were constructed to allow the students to work with both a familiar and an unfamiliar area of the world. The models were created using the method introduced 
by Djavaherpour, Mahdavi-Amiri, and Samavati (2017), which show the curvature of the Earth in each area's particular spatial extent, resembling a piece of a large globe. The first model, with 9 rhombus cells, covered eastern Australia, and the second model covered western Canada with 16 cells (Fig. 2). It is important to note that the cell surfaces are smooth and they do not express surface topography or thematic information like a 3D tactile globe or map would.

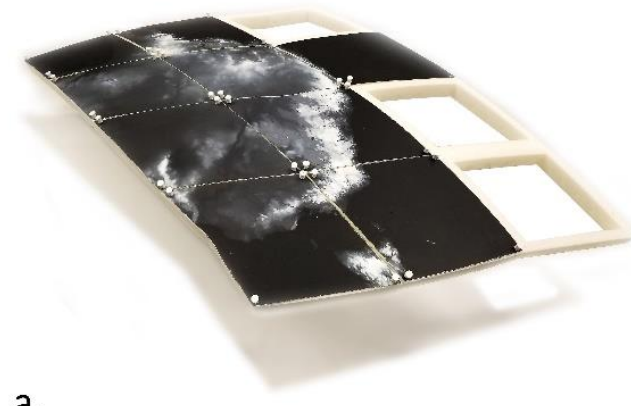

a.

Figure 2. (a) The physical model of Eastern Australia with elevation data attached to it $(55 \mathrm{~cm} \times 40 \mathrm{~cm})$. (b) The physical model of Western Canada with elevation and temperature $(55 \mathrm{~cm} \times 55 \mathrm{~cm})$.

Datasets were retrieved, printed on transparent media, and attached to the models via a pin-hole design, which supports attachment of several layers of overlaid data, as well as basic interactions such as sketching (Fig. 3). For the purpose of the study, the layers on the model were specifically ordered on the model with best visibility of the data in mind.

The opaque base layer of the model consisted of shaded elevation data presented with a grayscale (black representing sea level and white representing the highest elevations) (Fig. 4a). Temperature, precipitation, and population density were printed as transparent colored layers to overlay (Fig. 4). Two different symbology options were used for the temperature and precipitation data. The first symbology was a single color representing the range of values. Higher values were represented as highly saturated color, while lower values were 
less saturated. On transparencies this resulted in high values looking bright, while lower values were increasingly transparent (Fig. 4b,c). A second symbology consisted of multiple colors representing the range of values (Fig. 4d,e). The population density was displayed as a multiple color heat map (Fig. 4f). These data were chosen as they have a broad appeal to different grades and were very relevant to the Grade 8 curriculum. Students worked with the data layers on the model and independently of the model, to ensure comments could be distinguished as to their relevance to the data layers alone or when on the model. Students were able to choose the order of the layers when the layers were off of the model.

a.

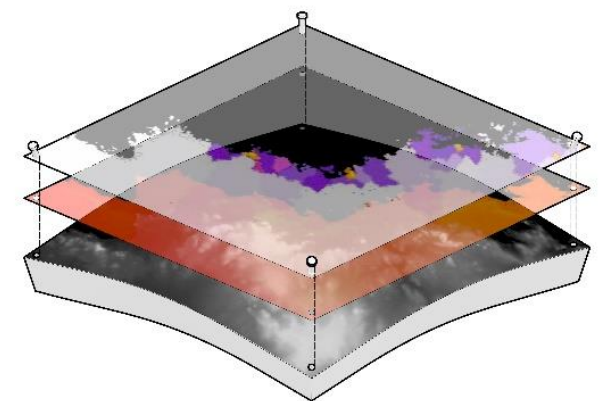

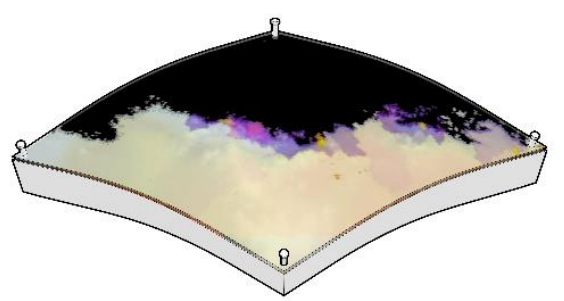

b.

Figure 3. (a) The pin-hole mechanism of the model. Flat layers of transparent data are printed in such way that they cover the curved surface of the model once attached to it. Pins then fix the layer in its place. Shown gray scale elevation, single color ramp temperature, and multicolor population density. (b) A model segment with three layers of data visible.

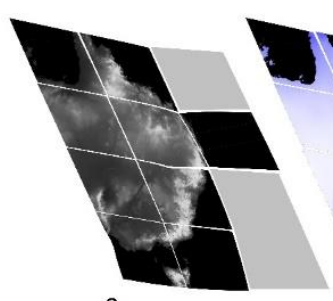

a.

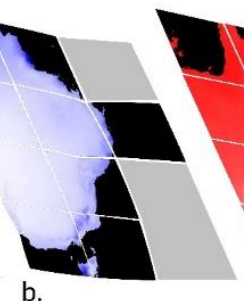

b.

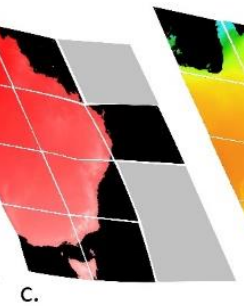

c.

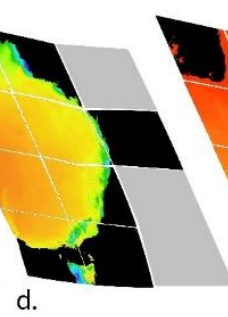

d.

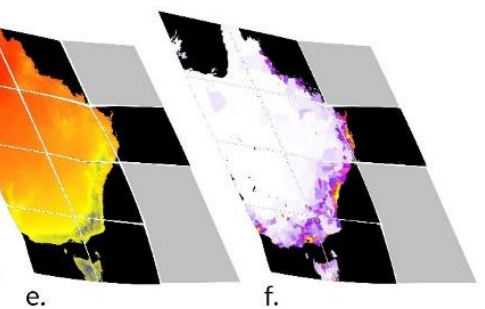

e.

Figure 4. (a) Gray scale 'elevation' data as base layer, (b) single color 'precipitation', (c) single color 'temperature', (d) multiple color 'precipitation', (e) multiple color 'temperature', (f) 'population' data. 


\section{Participants}

Participants in the study were 68 students from four classrooms within three different schools in Brisbane, Australia. The students were predominantly 13-year-old females in Grade 8, although participants also included thirteen female Grade 10 students and six male Grade 11 students (Fig. 5). The scope of this study was exploratory, to capture student experience, and thus it was felt important to not bind the study to the dominant demographic group (13-yearold, Grade 8 females), but include all potential participants. The analysis, therefore, takes into account all participants and does not analyze findings by gender or age given the uneven distribution of the sample of students. The schools are private schools, and while a high proportion of Brisbane secondary students attend private schools (Clun, 2017), this may also present limitations in socio-economic representation. This limitation is not critical to this study, but is important for context and should be addressed in future work.

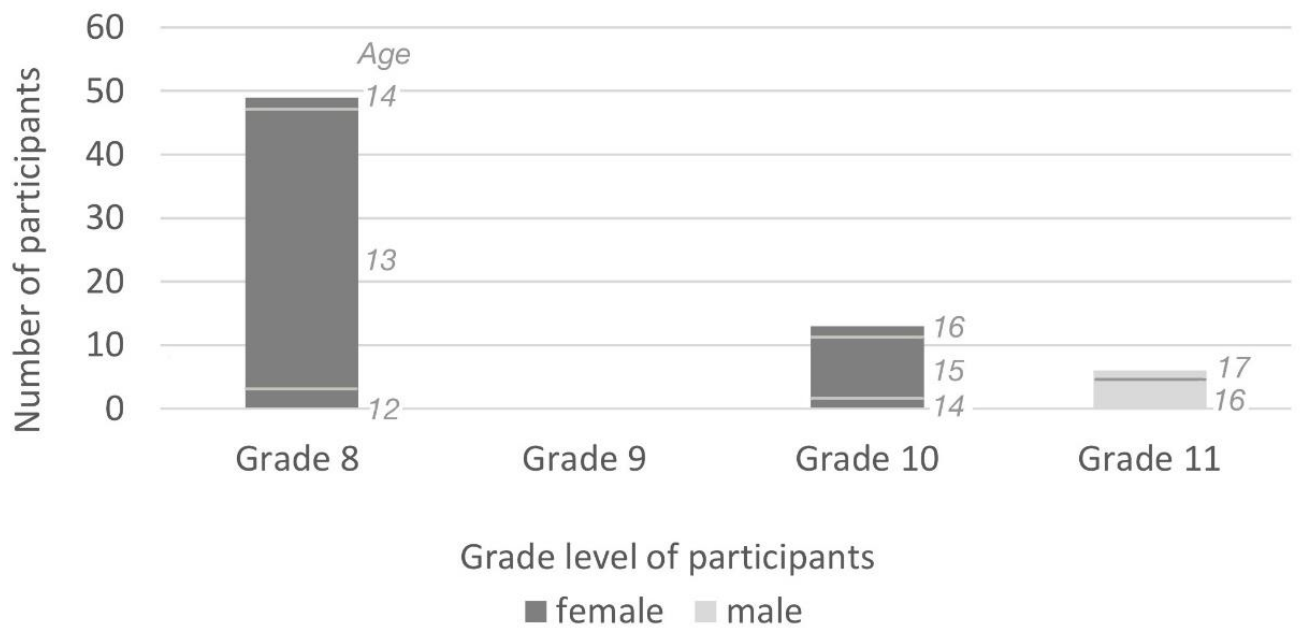

Figure 5. Participant numbers and distribution of grade and gender. Participant schools were single gender schools, resulting in all male or all female classes. Age distribution of participants in each grade is shown by numbers in italics to the right of the columns. 
All participating students were in geography classes, and their teachers were trained geography educators. Permission to conduct research in each class was given by both the teacher and principal of the school, and student participation required permission from the students themselves and their parent/guardian. This age range was selected because the students already had exposure to geographic representations and were familiar with small scale representations of Australia, enabling them to describe locations. At this level of education (Grade 8 and higher), Australian students are expected to be able to read geographical data and express spatial patterns and trends, and begin to look at multivariable data. In the Grade 8 national geography curriculum, outcomes include developing skills to interpret geographical data and other information using qualitative and quantitative methods,

and digital and spatial technologies as appropriate, to identify and propose explanations for spatial distributions, patterns and trends, and infer relationships (Australian Curriculum, Assessment and Reporting Authority n.d.). By Grade 10 in the Australian national curriculum, students should be able to identify how GIS might be used to analyze geographical data and make predictions (Australian Curriculum, Assessment and Reporting Authority n.d). Individual participants are referred to in this paper as "P" followed by a participant numeric code (e.g., P36).

\section{User Study}

Pre-test

The students completed a survey focused on questions about basic student demographics, their personal travelling experience, technology experience and skills, and geography selfefficacy. A written pre-test was conducted to ascertain students' basic geographic vocabulary 
and grasp of concepts to serve as a baseline for the subsequent Earth model exercises, including cardinal directions and querying between basic maps. Then the students rotated between stations where the layers of data were presented both on and off the models, and were asked questions and to complete specific data interpretation tasks.

\section{Tasks}

The tasks for the pre-test (paper), and for the map layers on and off the models, were focused on reading data, analyzing data and data relationships (including examining relationships between two or three layers of data), map projection, and applying the data to answer a geographic question:

- On the paper task, a simple grid of four squares was used as a map, with an attribute value given to each square (Fig. 6)

o Participants were asked questions relating to identifying the attributes of a certain square, or finding a square with certain attributes (Fig. 6).

- Reading data on the thematic layers and model consisted of:

- Identifying the values displayed by a DGGS cell,

- Finding the cell or area with the maximum or minimum value for the whole dataset, and

- Identifying ranges of values (Fig. 7).

- The map projection task asked the participants to show the extent of the area covered by the models on a paper map of that region, and then to explain why the shape of the areal extent was different between the model and the map.

- Analyzing data included: 
Determining trends in data (e.g., latitude and temperature, determining direction of water flow with elevation data),

- Identifying areas that met conditions of multiple criteria (high population density and low temperature and high precipitation),

- Expressing relationships within single datasets (e.g., trends in temperature), and between multiple criteria (e.g., determining which data correlated best with population density, and determining the relationship between elevation and temperature values).

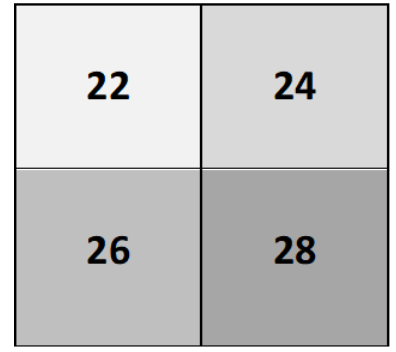

Average high temperature in degrees Celsius

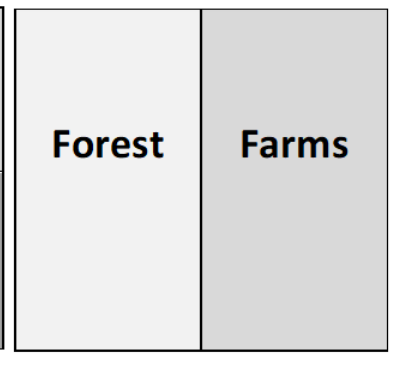

Land use/Land cover

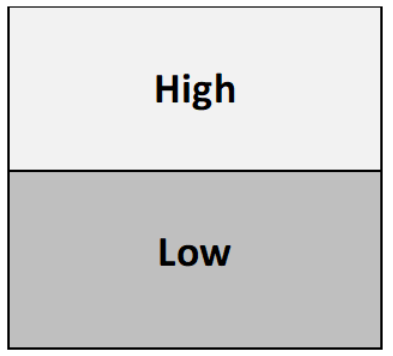

Precipitation

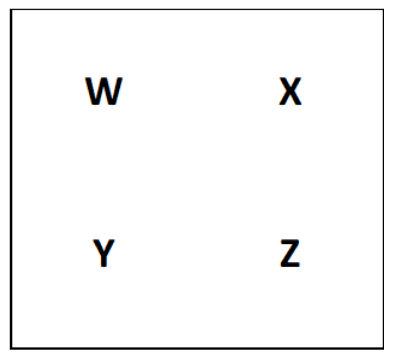

Reference points

Figure 6. Tasks on the paper test focused on the idea of three layers of data to represent the same area. Questions asked about attribute values at certain reference point locations and asked which location met certain multiple criteria.
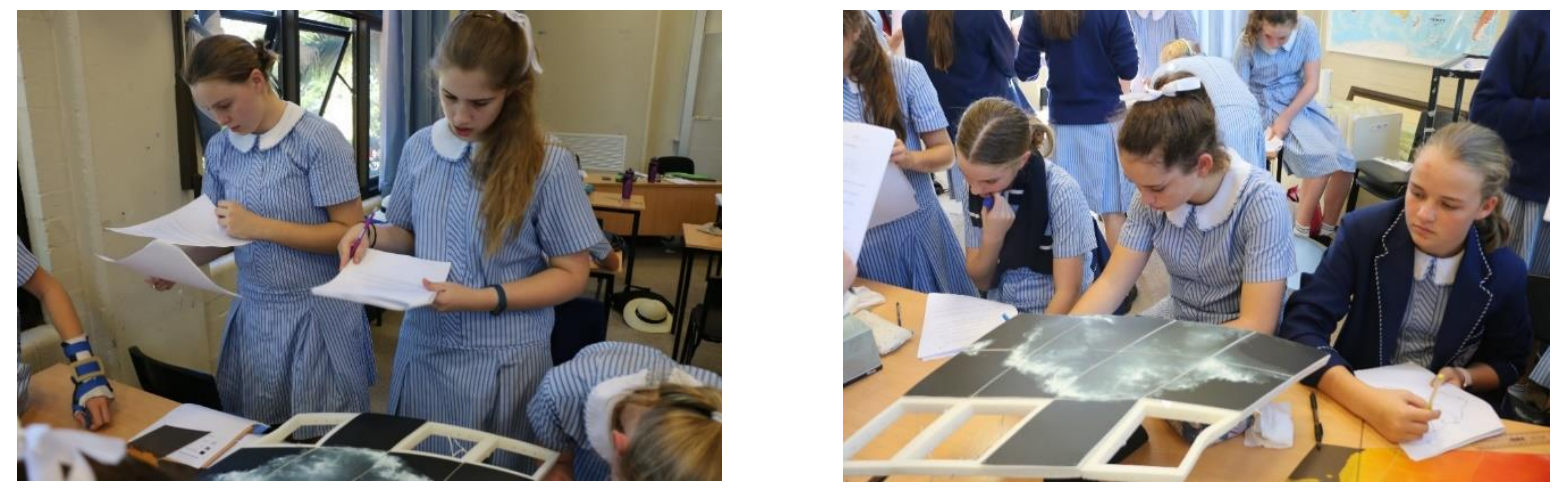

Figure 7. Participants worked on the models in their classroom environment. 
The students were asked to extract information from one, two, and three layers, and were also tasked with extracting information from single color shading and also multiple colored symbology.

The participants were asked for their preferences on how the data layers were presented (on the model or flat [i.e., off the model]), how symbology choices affected the readability of the data, their own self-efficacy in determining relationships in multiple datasets, whether they liked to learn with the model or not, whether the curve in the model was useful in building understanding of the data itself, strategies for using the model, improvements they would like to see, and whether they could see using the model with other types of data or in other classes.

\section{Analysis}

The paper-based geographic knowledge and skills tasks were analyzed for correct results, as were the layer and model exercises. Participants were also asked for feedback about using the model, and rich information was provided in the responses. Qualitative analysis of the feedback comments was used as it can enable an elicitation of the participant experience, through their own voice to provide a thorough description and insight into a phenomenon (Merriam 1998). The qualitative inquiry also relies on multiple representation of the experience, and in this case, observations, results of participant tasks, and participant feedback were used to aid in the validation of the data and credibility of interpretation. A qualitative thematic analysis was conducted to distill the essence of the feedback responses using a frame and code analysis (Goffman 1974). For each open-ended question, the responses were all examined, common themes that naturally emerged from the data were identified and quotes captured to allow for the authentic participant voice to be heard. The 
goal of the qualitative approach of this part of the study (aimed at addressing the second research question) was to gain insight to guide future practice (Merriam 1998) and inform further development of physical geospatial models in the classroom.

\section{Results}

\section{Reading data}

Results on the paper-based tasks indicated the students had strong geography fundamentals and could identify cardinal directions, understood the concept of scale with a graphic scale bar, could read data from a single dataset, and identify areas through multiple criteria, fundamental to the overlay process (Table 1). It is important to note these data tasks measured the basic overlay process, using letters and numbers for attributes, not shading or colors of the areas as represented in a choropleth map or raster images. The purpose of this basic exercise was to ensure question wording was effective and the students could spatially compare areas with multiple attributes and select areas that meet certain attribute criteria, which was confirmed.

Table 1. Participants' performance in paper-based tasks. Values indicate the percentage of students completing the task correctly.

\begin{tabular}{|l|c|}
\hline Tasks & \% correct \\
\hline Direction & 98 \\
\hline 4 point compass & 89 \\
\hline 8 point compass & 83 \\
\hline Scale & \\
\hline Determine distance from scale bar & 94 \\
\hline Multi-criteria Overlay & 94 \\
\hline Match criteria, find location & 82 \\
\hline Match criteria, find location & 87 \\
\hline Find location (with no area matched) & \\
\hline Describe attributes of location &
\end{tabular}


Reading data layers on the model, participants showed competence in answering all questions relating to the data reading of a single variable, and were better interpreting multicolored data than interpreting shades of a single tone. The exception occurred in identifying a range of data, in which the single color interpretation had more accurate results. There was also confusion among $10 \%$ of the participants of how multiple colors could be used to represent values of just one variable, thinking that the multiple colors represented multiple datasets. Participants were able to analyze relationships between two layers quite easily, but struggled more when three layers were considered. The results of this part of the study are presented in Figure 8.

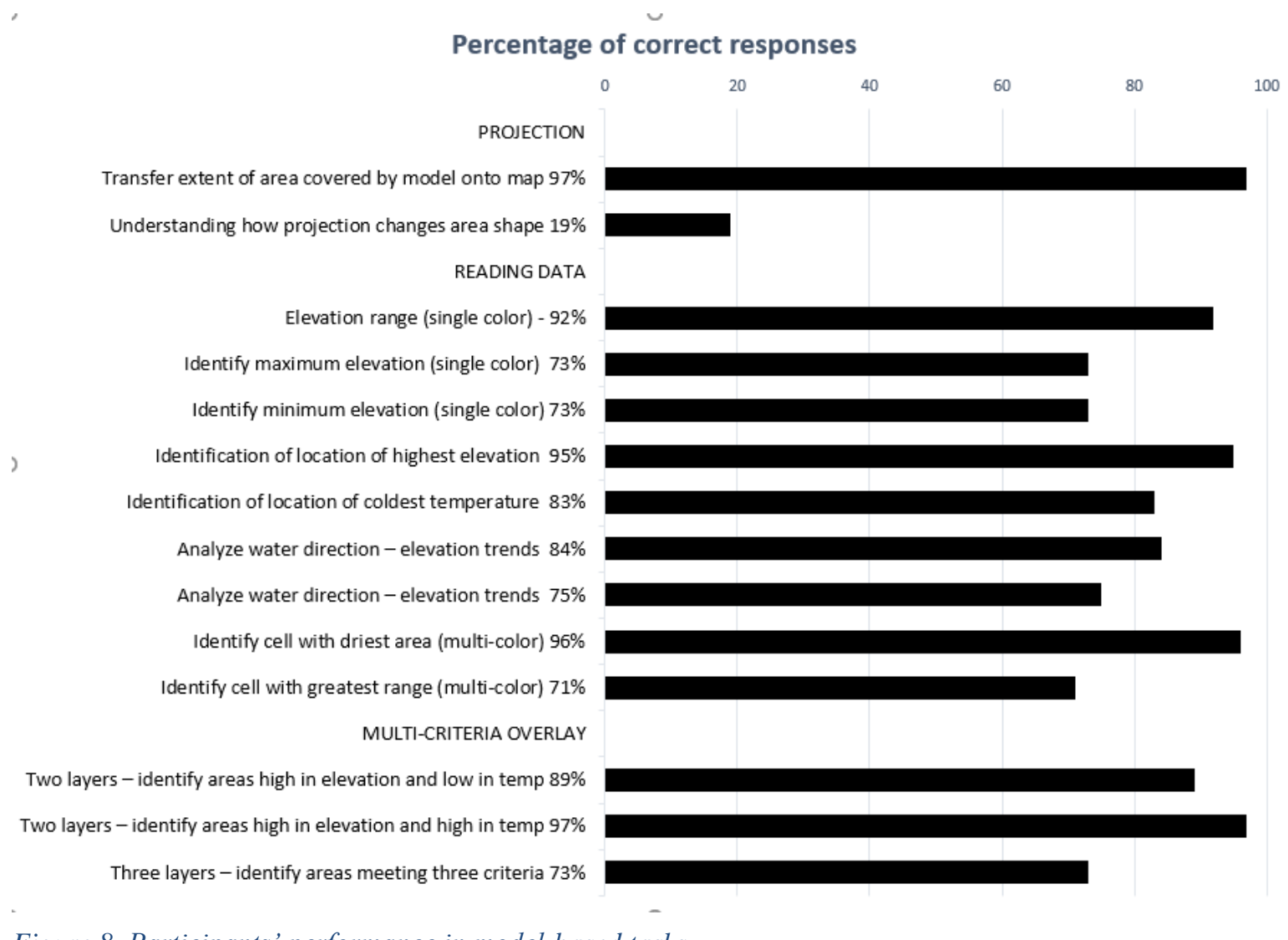

Figure 8. Participants' performance in model-based tasks. 
A surprising finding resulted from the projection task. While students could easily define the extent of the area shown in the models on a paper map, only $19 \%$ could explain why the shape of the area differed between the model and the paper. Examples of inaccurate explanations of the difference due to projection distortion included "the rectangle is different because the land is always changing" (P9) and "the height of the elevation changes the shape as it is curved" (P20). Accurate descriptions never used the word "projection," but were clear in the explanation that the shape of a consistent area shown on both a curved surface and flat paper would be expressed differently due to distortion moving from a 3D to $2 \mathrm{D}$ surface. Participant 16 explained "because on the model the shape is slightly curved like the Earth and the drawing is flat." Participant 31 mentioned the dimensional transformation from a sphere to paper more specifically, "This is more exact to the earth. It is slightly rounded at the top because that is how the earth would be. You can't make a map on paper 3D."

\section{Feedback on Using the Model}

Students were asked about their preferences in reading the data on the model, particularly conducting an overlay analysis. The first question asked if it was easier to compare two layers when they were separated and visualized side-by-side on a table or overlaid on the model. Respondents answered that they preferred to overlay the layers on the model (62\%) or had no preference $(8 \%)$. The stated benefit of this approach was being able to see the layers simultaneously for easier visual comparison and analysis. When asked about preferences for three layers, $42 \%$ stated they preferred overlaying them, primarily for efficiency, and $54 \%$ stated that they found it easier to spread the layers out and look at them individually so they didn't get confused by the colors. 
Of all participants, $88 \%$ responded that they liked to learn with the model and $3 \%$ had no preference or did not answer. A few participants $(4.5 \%)$ indicated they had troubles understanding the data itself (one was color blind), leaving only one (1.5\%) who preferred to work with layers off the model.

When asked how the model helped in understanding the data (multiple responses were allowed), nearly half of the participants (44\%) indicated the realism portrayed by the model helped in their learning, and 37\% claimed they found it interesting to work with (Fig. 9). The findings were echoed in the thematic analysis of open comments with this question, with three main themes evident in if and how the model supported learning - realism, ease of use, interest - and one minor theme, fun.

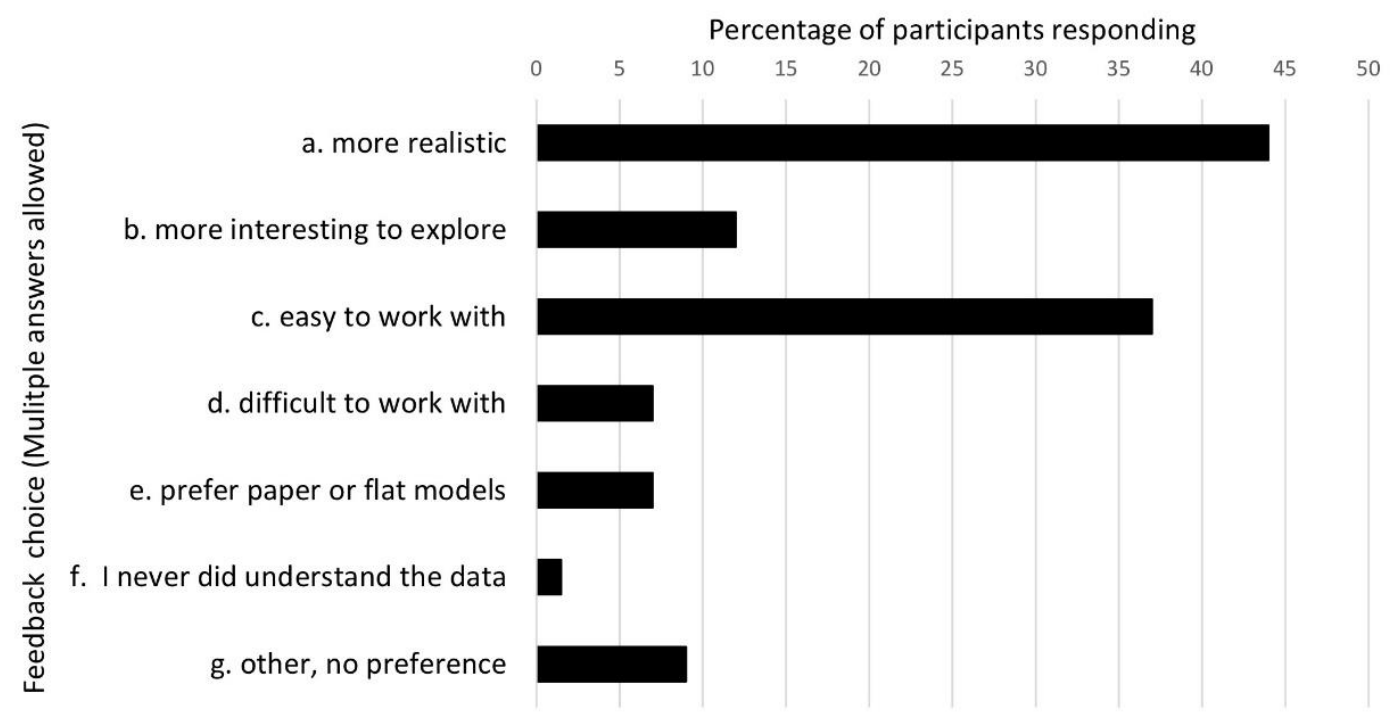

Figure 9. Participant responses to the question of if and how the model helped in understanding the data.

The importance of the realism of the model was emphasized in comments including "(the model was) more realistic, as Earth is a sphere (round) it was easier to understand" (P54), it "relates more to the real Earth" (P16). Some related the model to other technologies 
they had used to study geographic concepts: "Compared to a normal map, the earth isn't flat, so we should be learning on realistic maps" (P39), "its like a non-electronic Google Earth" (P22) and it was "more realistic and made more sense than Google Earth" (P27). Seven percent of students found the model difficult to work with, however, it was interesting to note that some of these participants still indicated they recognized its value in providing a realistic model of the Earth to help with learning.

The comments stating the model was easy to use were often tied to how participants liked to learn: "it was visual and helped me understand the information" (P24), "I'm a slow learner" (P37), "I'm a visual learner" (P7 and P18), "It is easier than just looking at google earth or a graph" (P23). The third theme focused on interest and novelty. Capturing the interest of K-12 participants is a goal of education, as increased interest can lead to better retention and time spent with the material (Ainley, Hidi, and Berndorff 2002), so this is an important theme for educators. Participants mentioned both the aesthetic nature of the model's appearance, and the fact that it was something new which provided interest. It was considered, "cooler, gets you to think more" (P46), "it gave the information in an intriguing and visually pleasing way." The fourth theme made explicit by the analysis was "no difference" (P64). The participants had no preference, or found no difference in understanding the data, whether on the model, or laid out flat. The fifth and final theme was that of "fun" - another important aspect of learning for this age group. "Fun" was stated in comments regarding the look of the model itself and also in the overlaid data. Comments included "It was more realistic which made it more interesting, this made it more fun to work with engaging me more" (P41), "It was more like earth and was more fun to work with" (P38), "It is more fun and looks kool (sic)" (P6), "the colors of temperature and elevation 
were easier and more fun to understand" (P35), and "It was fun to see the different elevation compared to temperature" (P26).

Other participants mentioned that the model was a catalyst to deeper thinking and collaborative learning, stating it was "clearer to look at with multiple people" (P43). There were two participants who were confused by the curvature, thinking that it represented the Earth surface relief, instead of shape. This was obvious in comments such as "the curve didn't match the shading in some areas" (P7) and "the top of the curve is higher and represents higher levels of land" (P13), and also from observing the participants bend to look at the profile of the model. These participants were reminded that the elevation was represented by shading and not shown by the model surface per se, but is an important reminder about assumptions made when giving students models and representations to work with.

Participants were asked about potential improvements to the model. The comments were relevant to the model itself and the data. For the model, participants wanted to see the incorporation of texture, or a 3D effect. Some wanted to see it larger, or show the rest of the Earth as a big globe. Along the same lines, some wanted to see the whole country and not just part of it on the model. Three percent of participants suggested making the model more interactive, and suggested a digital version, but did not mention GIS explicitly. The remaining comments focused on the data itself. Reference features were requested (8\%) to help in interpreting locations. Improvements suggested included better defining the ocean/land interface, showing landmarks and cities, and providing reference information such as a compass rose for directions. A small percentage (4\%) suggested improving the legend or integrating it into the model surface.

The participants were asked if they could see using this type of model in other areas of learning. They were challenged to extend the applications of the model beyond what they 
experienced, but some participants did state that they thought it would be useful in helping to understand abstract concepts in geography and environmental studies because "we can understand what it really looks like and that it's not just an idea" (P52). Some also acknowledged that they don't use many maps because "most subjects are humanities-based"

(P4), but other participants saw they could use the model to show "high and low poverty areas, ethnic diversity between cities/states/countries, tracking spread of infections/disease across a country, showing differences between the hungry and the well off and how that correlates with healthy/wealthy and non-healthy/non-wealthy areas" (P2).

There were two issues in handling the model that occurred while conducting the research in classrooms. The first was that it took a long time to set up the model and then place the data transparencies on each cell. The second was that the pins holding the cells together often broke, and the model needed to be a lot more robust in the classroom and while moving between classrooms. Wires were temporarily used in the place of the rigid pins to attach the cells. This allowed for a little movement of the cells without them coming apart, and to more easily pick up and move the model as a single entity. The transparent overlays were attached with invisible tape along their margins, and were draped over the model, and secured along the far edges of the model. This allowed the overlays to still fit exactly over the cells. Solutions to address these challenges are described in the discussion section.

\section{Discussion}

\section{Did the Model Support Learning of Geographic Concepts?}

The participants were able to understand and transfer information from the model to their paper maps. They could determine the area covered by the model and correctly represent the 
same area on their map. Very few students, however, were able to describe why the area was a different shape on the map (19\%; Figure 8), indicating that they were not aware of the projection distortions inherent in 2D maps, and perhaps could not appreciate the benefit of using a true-geometry-Earth model. This finding has implications for how students understand maps, but also how precise and accurate to the Earth's surface the model needs to be. In the current model, the shape of each cell is unique and exact to that specific location. For educational purposes, and at this scale, having a one-cell model that represents the curvature of the Earth with less precision, and can be overlaid with data from any area of the globe may be sufficient. This would also increase the usefulness of the model as a teaching resource.

The realism provided by the curvature of the model, however, was important to the learners in reflecting the true surface of the Earth. The participants indicated how important it was to them to use realistic models in their learning. Having another representation of Earth, especially one that looks different than other representations used in classrooms is important to dispel reified thinking of the world in a single projection form, with its inherent distortions, and to construct more robust mental maps of the world. Another element that helps in learning is the visual appeal of the model to this population, which attracted and retained participants' interest in using the model and time spent learning.

The participants were faced with interpreting data from both single color (shaded dark to light) and multi-colored layers. They generally performed better with, and preferred using, multiple colors to determine values in the data, with the exception of determining the range of values in a dataset. One participant however, showed a level of expertise in preferring the single color as they found the interpretation was far more intuitive and efficient with shades 
of gray, because they didn't need to keep referring to the key, as they did with the multiple colors.

The paper pre-test tasks confirmed that the participants were able to answer questions with geographic compass directions and could do a basic overlay analysis with three different layers. They were also able to do this analysis with the overlay transparencies, both on and off of the model for two layers, however, they were not as successful with three layers (layer 1: elevation in shades of gray, layer 2: precipitation with single color, layer 3: population density, sparse with colored polygons so all layers were visible). The success with the two layers (89\% and $97 \%)$ and moderate success $(73 \%)$ with the three layers suggests that the model is a good bridge between maps and digital technology, or GIS. A few participants even suggested that that a digital form and interactivity with the model would be useful, and in effect, they were describing a GIS. Other participants suggested the model resembles an analogue (non-digital) Google Earth but was more realistic and made more sense than the software.

The participants indicated that they learned geographic content knowledge about both the familiar (Australia) and unfamiliar places (Canada). The models also facilitated a consistent scale comparison of the two countries. This is not feasible with DE applications as the two countries cannot be seen on a single hemisphere view at the same time. The participants also mentioned gaining process knowledge by learning how to overlay multiple data layers on one model.

For a few participants, there was confusion around what the curvature of the model represented. Comments made by these participants in the feedback and tasks and researcher's observations of their use of the model made it clear they thought the curvature of the model represented the relief of the Earth's surface. These few students studied the profile of the 
model and one mentioned how the curvature didn't align with the what they knew about the elevation in an area. To mitigate confusion that might arise from students thinking from the shape of the model mimics the terrain relief, it is important to have the model described to the participants beforehand (what it shows and what it doesn't show). Moorman and Crichton (2018) suggested the need for a similar approach with software (Google Earth), as students benefit from a technology knowledge framework to support their subsequent content learning from any technology.

\section{The Student Experience with the Model}

A strong majority (88\%) of the participants liked working with the model, and even the ones who didn't or who were impartial, indicated they saw value in using it. The model was described as easy to use, novel and fun, which provided more interest, aligning with the findings from Gray et al. (2011) in their study of hands-on physical models in Earth Science education. Interest is an important element of learning to keep students engaged and to secure their learning. The model also helped participants to become interested in and learn about unfamiliar places, especially when comparisons with known places were enabled.

The colorful data attracted more attention and was more appealing to this audience than shades of gray that were used as the base elevation data. In order to make the data even more useful, more reference data are required, including borders and settlements and tools such as a compass rose. An important observation by a participant revolved around the idea of collaborative learning and the benefit of having multiple students looking at the one model at once. Research observations confirmed that students were gathered around the model, all having a chance to view it from their own perspectives. In a DE or GIS, there is only one perspective at a time that is controlled by one person. In this case, the students were 
completing the tasks independently, but they were still able to see the potential for collaboration. This finding also aligns with observations from model use in classrooms in Gray et al. (2011).

\section{Classroom suitability}

The students made helpful suggestions about improving the model, including making the model bigger and showing whole countries at once, adding textural features and 3D effects. A solution to represent a three-dimensional elevation is to use Augmented Reality (AR) to add a virtual height to the physical flat model. Such an approach directs the future work for this research, as well as another study to evaluate the effectiveness of various methods to create 3D elevation layers and support data overlays.
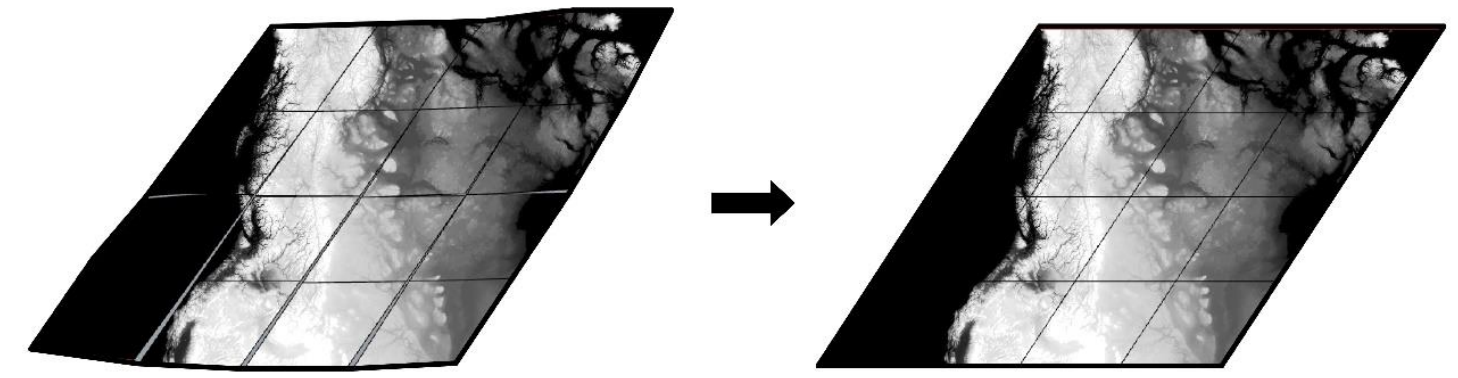

Figure 10. Converting the original DGGS-based geometry to one rhombus made up of 16 uniform cells.

Being able to $3 \mathrm{D}$ print the cells and ink print the layers on transparencies makes the model a cost-feasible resource for K-12 education. From observations in the classroom, the model needs to be more robust and the time to put it together shortened if it is to be useful for busy teachers and quick paced class environments. To address this issue, the geometry of cells can be slightly modified to make them uniform and easier to put together than the customized shape of each cell required to preserve the fidelity of the geoid. This would result 
in one model to host any part of the Earth instead of custom models. Such a distortion of the original cells does not impact the correspondence between data layers and the underlying cells (Fig. 10).

Since a Digital Earth system is used for data retrieval purposes, each cell and its corresponding datasets are identified by a unique DGGS index. DGGS indexing methods are developed to efficiently address common tasks (e.g. data retrieval, hierarchical access, and data compression) in the resulting Digital Earth. Using these indices for distinguishing different physical cells may complicate the assembly task in the classroom. One possible solution is to map the original DGGS indexing to a simpler set of indices. Fig. 11 shows our proposed mapping, which uses a simple combination of the letters and numbers.

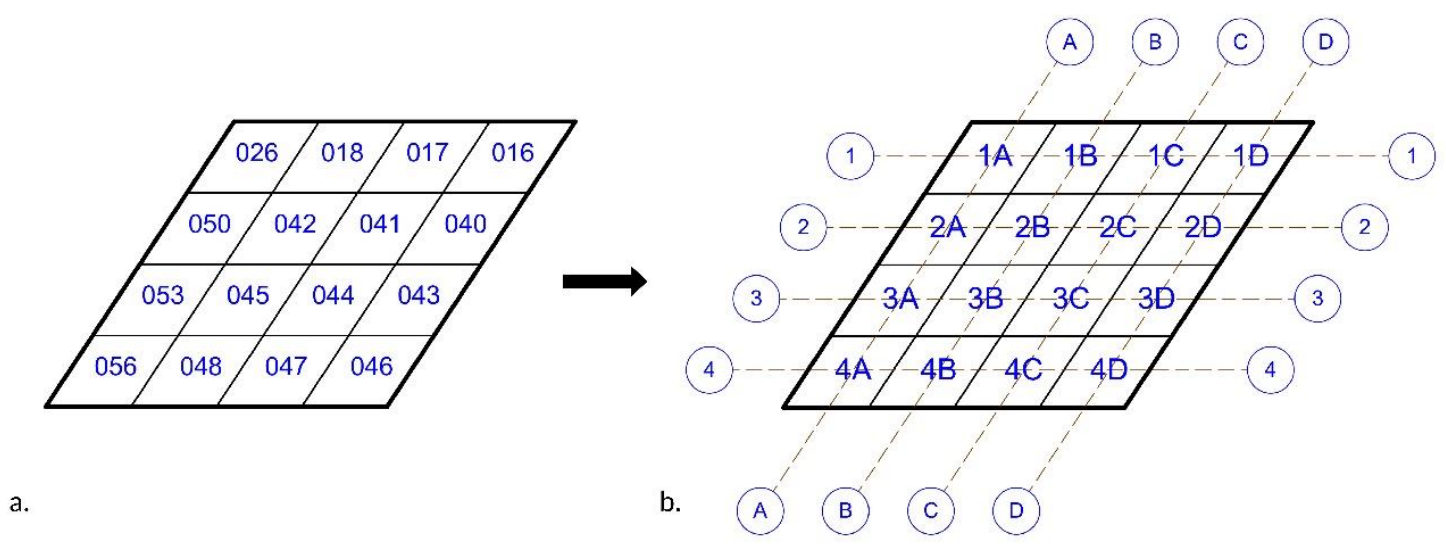

Figure 11. (a) The previously used DGGS-based method. (b) The proposed method of indexing the cells.

To address the breakage issue of the plastic pins, the attachment details of the cells were redesigned with a pair of screws and nuts for each edge of the cells. Screws are easy to drive, the precision of the 3D printer does not affect them as opposed to the alignment required for the plastic connectors, and they structurally support the whole model once all the rhombus cells are attached (Fig. 12). 


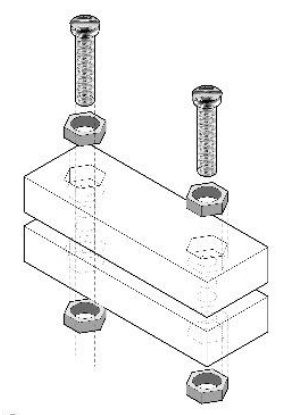

a.

b.

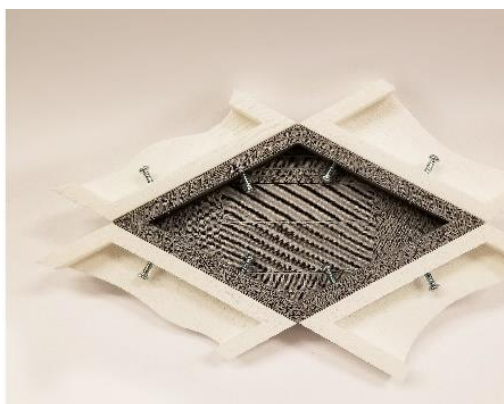

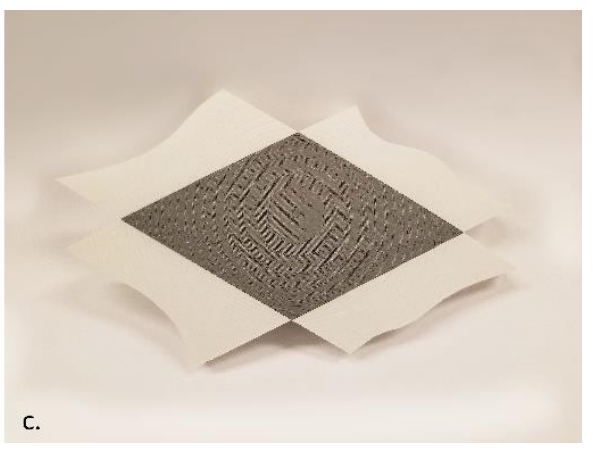

Figure 12. (a) Detail of the redesigned assembly method on the edge of the rhombuses. (b) Shown from below, detail of the redesigned assembly. (c) Shown from above, the redesigned connection method of the rhombuses using screws and nuts. It is fast to assemble and disassemble, does not break, and structurally supports the whole model.

\section{Conclusion and Future Work}

In this work we explored the intersection of geography education and physicalization, and put a physical geospatial model with removable transparent datasets in the hands of 68 school children. We asked three questions: 1) How did the students learn best with the model, particularly in the construct of overlay? 2) What was the student experience using the model? And 3) How well suited is the model to a K-12 classroom?

To address these questions, a mixed-methods study was conducted involving student participants in their own classrooms using authentic data that tied to their curriculum outcomes. The participants completed geographic tasks on paper, and with the model, and also provided feedback about their experience using the model. Tasks included reading data and conducting analyses, particularly through the concept of overlay - layering spatially referenced data and examining the relationships of all layers. The physical model not only provided a unique "view" of the world, it also allowed a deconstruction of the requisite elements of a student's learning progression for generating meaning with geospatial representations. The model supported geographic learning by acting as an analogue GIS, allowing participants to manipulate and see the data and associated relationships. Participants 
were successful in exercises where two layers were combined and analyzed. Three layers provided more challenge, as did a single-color palette vs. multi-color.

From an educational perspective, the model was beneficial as a learning tool as it created interest and engagement among the participants, which was also reiterated in the participant feedback. Participants mentioned that they want to work with representations that are accurate to the shape of the Earth and appreciated the correct curvature of the model. Using authentic data in the natural learning environment is also a goal of $21^{\text {st }}$ century education that is supported by the model. Themes that emerged through a frame and coding analysis of the feedback responses included the model being realistic, easy to use, interesting, and fun.

Given this positive response, future work includes creating models with the improvements suggested in our discussion, and building educational resources to support different applications of the models and overlays, including better enabling students to independently choose and reorganize the layers. We will explore the incorporation of Augmented Reality (AR) to add a virtual height to the physical flat model for terrain or 3D attribute mapping, and also physical terrain relief representation in the realm of hyper and tactile globes. Our study was limited in class time available and access to students whom represented a narrow socio-demographic profile, including a narrow age range and nearly singular gender representation. Future research includes broadening the scope of the study's participant demographics and analyzing findings based on gender or age-related differences, to better understand how and in what grades the models can best be used to address geography education goals.

Our study has provided an insight into a population of young people in their natural learning environment and their preferences for geovisualizations for learning. This work 
aligns with geography education research priorities around the world and will ultimately inform pedagogical strategies to critically use geospatial datasets in K-12 schools and future model/physicalization development to facilitate geographic learning.

Acknowledgements:

The authors are indebted to student research assistant Kendra Garbutt who compiled and organized the data. This work was supported by a Social Science and Humanities Research Council (SSHRC) Insight Development Grant (430-2016-00784) to L. Moorman and a Natural Science and Engineering Council (NSERC) CRD Grant (CRDP) 477150-14 grant to F. Samavati. Ethics was approved by Human Research Ethics Board (HREB) Certificate \# 100937 at Mount Royal University. Many thanks to Queensland University of Technology for supporting the work in Australia and the participating schools, students and teachers; and to Global Grid Systems (formerly Pyxis Innovation) for the sponsorship of the NSERC CRD.

\section{References}

Ainley, M., S. Hidi, and D. Berndorff. 2002. Interest, learning, and the psychological processes that mediate their relationship. Journal of Educational Psychology 94(3): $545-561$.

Alderson, T., M. Purss, X. Du, A. Mahdavi-Amiri, and F. F. Samavati. 2020. Digital Earth platforms. In Manual of Digital Earth, ed. H. Guo, M. F. Goodchild, and A. Annoni, 25-54. Singapore: Springer Nature.

Australian Curriculum. Assessment and Reporting Authority. N.d. Geography aims year 10. Accessed February 14, 2020. Australian Curriculum Website

Baker, T.R. 2002. The effects of Geographic Information System (GIS) technologies on students' attitudes, self-efficacy, and achievement in middle school science classrooms. University of Kansas.

Baker, T.R., S. Battersby, S.W. Bednarz, A. M. Bodzin, B. Kolvoord, S. Moore, D. Sinton, and D. Uttal. 2015. A research agenda for geospatial technologies and learning. Journal of Geography 114(3): 118-130. 
Bearman, N., N. Jones, I. André, H. A. Cachinho, and M. DeMers. 2016. The future role of GIS education in creating critical spatial thinkers. Journal of Geography in Higher Education 40(3): 394-408.

Bednarz, S., S. Heffron, and N. Huynh. 2013. A road map for 21st century geography education: Geography education research (A Report from the Geography Education Research Committee of the Road Map for 21st Century Geography Education Project). Washington, DC: Association of American Geographers.

Clun, R. The Brisbane suburbs where nine in 10 students go to private schools. Brisbane Times. Last Modified November 7, 2017. Accessed August 20, 2020. https://www.brisbanetimes.com.au/national/queensland/the-brisbane-suburbswhere-nine-in-10-students-go-to-private-schools-20171107-p4ywvk.html

Dadkhahfard, S., K. Etemad, J. Brosz, and F. F. Samavati. 2018. Area preserving dynamic geospatial visualization on physical globe. VISIGRAPP 3: 309-318.

Djavaherpour, H., A. Mahdavi-Amiri, and F. F. Samavati. 2017. Physical visualization of geospatial datasets. IEEE Computer Graphics and Applications 37(3): 61-69.

Gallagher, S. M. and R. M. Downs. 2012. Geography for life: National geography standards. National Council for Geographic Education.

Goffman, E. 1974. Frame analysis: An essay on the organization of experience. Harvard University Press.

Golledge, R.G., M. Marsh, and S. Battersby. 2008. Matching geospatial concepts with geographic educational needs. Geographical Research 46(1): 85-98.

Gray, K.R., K. D. Owens, D. N. Steer, D. A. McConnell, and C. C. Knight. 2011. An exploratory study using hands-on physical models in a large introductory Earth Science classroom: Student attitudes and lessons learned. Electronic Journal of Science Education 12(2): 1-23.

Greco, S.E. 2018. Seven possible states of geospatial data with respect to map projection and definition: A novel pedagogical device for GIS education. Geo-spatial Information Science 21(4): 288-293.

Horowitz, S. S. and P. H. Schultz. 2014. Printing space: Using 3D printing of digital terrain models in geosciences education and research. Journal of Geoscience Education 62(1): 138-145. 
Jansen, Y. and P. Dragicevic. 2013. An interaction model for visualizations beyond the desktop. IEEE Transactions on Visualization and Computer Graphics 19(12): 23962405.

Jansen, Y., P. Dragicevic, and J. D. Fekete. 2013. Evaluating the efficiency of physical visualizations. In Proceedings of the SIGCHI Conference on Human Factors in Computing Systems. 2593-2602. New York: ACM Press.

Jansen, Y., P. Dragicevic, P. Isenberg, J. Alexander, A. Karnik, J. Kildal, S. Subramanian, and K. Hornbæk. 2015. Opportunities and challenges for data physicalization. In Proceedings of the 33rd Annual ACM Conference on Human Factors in Computing Systems. 3227-3236. New York: ACM Press.

Kastens, K.A. and A. Rivet. 2008. Multiple modes of inquiry in earth science. The Science Teacher 75(1): 26-31.

Kastens, K. A. and A. Rivet. 2010. Using analogical mapping to assess the affordances of scale models used in earth and environmental science education. Spatial Cognition 2010. Lecture Notes in Computer Science 6222: 112-124. Springer, Berlin, Heidelberg.

Liben, L. S. and R. M. Downs. 1989. Understanding maps as symbols: The development of map concepts in children. Advances in child development and behavior 22: 145201. JAI.

Mahdavi-Amiri, A., F. F. Samavati, and P. Peterson. 2015. Categorization and conversions for indexing methods of Discrete Global Grid Systems. ISPRS International Journal of Geo-Information 4(1): 320-336.

Mahdavi-Amiri. A., T. Alderson, and F. F. Samavati. 2015. A survey of Digital Earth. Computers and Graphics 53: 95-117.

Merriam, S. B. 1998. Qualitative research and case study applications in education. Revised and expanded from" Case study research in education.". San Francisco, CA: Jossey-Bass Publishers.

Mishra, P., \& Koehler, M. J. 2006. Technological pedagogical content knowledge: a framework for teacher knowledge. Teachers College Record, 108 (6), 1017-1053. 
Moorman, L. A. and S. Crichton. 2018. Learner requirements and geospatial literacy challenges for making meaning with Google Earth. International Journal of Geospatial and Environmental Research 5(3): Article 5.

Rivet, A. E. and K. A. Kastens. 2012. Developing a construct-based assessment to examine students' analogical reasoning around physical models in Earth Science. Journal of Research in Science Teaching 49(6): 713-743.

Stusak, S., J. Schwarz, and A. Butz. 2015. Evaluating the memorability of physical visualizations. In Proceedings of the 33rd Annual ACM Conference on Human Factors in Computing Systems. 3247-3250. New York: ACM Press.

Stusak, S., M. Hobe, and A. Butz. 2016. If your mind can grasp it, your hands will help. In Proceedings of the TEI'16: Tenth International Conference on Tangible, Embedded, and Embodied Interaction. 92-99. New York: ACM Press.

Taher, F., J. Hardy, A. Karnik, C. Weichel, Y. Jansen, K. Hornbæk, and J. Alexander. 2015. Exploring interactions with physically dynamic bar charts. In Proceedings of the 33rd Annual ACM Conference on Human Factors in Computing Systems. 32373246. New York: ACM Press. 